\title{
Digitalisierung personennaher Dienstleistungen in der Kinder- und Jugendhilfe: Entwurf und Evaluation eines Informationssystemdesigns
}

\author{
Michael Fellmann • Birger Lantow • Gregor Simon
}

Eingegangen: 11. April 2020 / Angenommen: 15. Juni 2020 / Online publiziert: 16. Juli 2020

(C) Der/die Autor(en) 2020

Zusammenfassung Personenbezogene Dienstleistungen in der Kinder- und Jugendhilfe stellen in Deutschland einen erheblichen Anteil der nachgefragten Dienstleistungen sowie einen bedeutenden Wirtschaftszweig dar. Sie zeichnen sich durch die Interaktion verschiedener Individuen innerhalb komplexer sozialer Prozesse aus. Dabei besteht im täglichen Arbeitsalltag die Herausforderung, schwach strukturierte Arbeitsprozesse, informelle Kommunikationsstrukturen sowie Erfahrungswissen so zu integrieren, dass einerseits eine bestmögliche persönliche Unterstützung der Klienten erreicht wird, andererseits individuelle und organisationale Arbeits- und Lernprozesse ermöglicht werden. Trotz der enormen Bedeutung der personenbezogenen Dienstleistungen existiert in diesem Bereich bisher keine adäquate Unterstützung der Arbeit durch dedizierte Informationssysteme, sodass vielfach auf Consumer-IT zurückgegriffen werden muss. Im vorliegenden Beitrag berichten wir daher über den Entwurf und die Evaluation eines branchenspezifischen Informationssystemdesigns zur Unterstützung personennaher Dienstleistungen im Bereich der Kinderund Jugendhilfe. Zunächst werden in diesem Bereich die aus mehreren Quellen gewonnenen und zusammengeführten Anforderungen überblickartig vorgestellt. Auf deren Grundlage wird schließlich die Gestaltung eines Informationssystems vollzogen. Der Systemvorschlag wird anschließend im Hinblick auf die geschaffenen Potenziale von Praktikern bewertet sowie einer kritischen Reflexion unterzogen. Abschließend werden Potenziale für die weitere Forschung vorgestellt.

\footnotetext{
M. Fellmann $(\varangle) \cdot$ B. Lantow $\cdot$ G. Simon

Lehrstuhl für Wirtschaftsinformatik, Universität Rostock, Rostock, Deutschland

E-Mail: michael.fellmann@uni-rostock.de

B. Lantow

E-Mail: birger.lantow @uni-rostock.de

G. Simon

E-Mail: gregor.simon@uni-rostock.de
} 
Schlüsselwörter Personennahe Dienstleistungen - Informationssystemgestaltung · Anforderungen · Use Cases · Kinder- und Jugendhilfe

\title{
Digitalisation of Person-Oriented Services in the Field of Child and Youth Welfare: Design and Evaluation of an Information System Design
}

\begin{abstract}
Personal services in child and youth welfare in Germany represent a considerable share of the services in demand and an important branch of the economy. They are characterised by the interaction of different individuals within complex social processes. In the daily work routine, the challenge is to integrate weakly structured work processes, informal communication structures and empirical knowledge in such a way that on the one hand the best possible personal support of the clients is achieved and on the other hand individual and organisational work and learning processes are enabled. Despite the enormous importance of personal services, there is no adequate support for work in this area by dedicated information systems, so that consumer IT must be used in many cases. In this paper we therefore report on the design and evaluation of a sector-specific information system design to support person-related services in the field of child and youth welfare. At first, existing requirements in this area are presented and the requirements gained from several sources are summarized. Based on these requirements, the design of an information system is finally carried out. The system proposal will then be evaluated by practitioners with regard to the created potential. Finally, potentials for further research are presented.
\end{abstract}

Keywords Personal-centric services - Information system design · Requirements · Use cases $\cdot$ Child and youth welfare

\section{Motivation und Einleitung}

Die Digitalisierung ist eines der bestimmenden Themen unserer Zeit. Informationsund Kommunikationstechnologien führen zu einem Wandel im gesellschaftlichen Leben (Schütte 2014). Neben einer Zunahme an digital gespeicherter Information und Verschmelzung von realer und virtueller Welt (vgl. Herterich et al. 2016) werden immer mehr Prozesse von Maschinen bzw. intelligenten IT-Systemen übernommen (Motahari-Nezhad und Swenson 2013). Diese Entwicklung führt zu grundlegenden Änderungen im Bereich der Produktion und Dienstleistung (Motahari-Nezhad und Swenson 2013), die für den Dienstleistungssektor besonders relevant sind, denn er besitzt den größten Anteil an Beschäftigten mit mehr als $70 \%$ (vgl. Bsirske 2014). Mit Ausgaben von über $48 \mathrm{Mrd}$. € im Jahr 2017 (Statista 2019) stellen insbesondere personennahe Dienstleistungen in der Kinder- und Jugendhilfe in Deutschland einen erheblichen Anteil der nachgefragten Dienstleistungen sowie einen bedeutenden Wirtschaftszweig dar. Dieser gewinnt zudem weiter an Bedeutung. So haben etwa die Kindeswohlgefährdungen 2018 um $10 \%$ auf 50.400 betroffene Kinder und Jugendliche zugenommen (Destatis 2019a). Im selben Jahr haben die Träger der Kinder- und Jugendhilfe in Deutschland mehr erzieherische Hilfen für Menschen 
unter 27 Jahren gewährt als jemals zuvor (Destatis 2019b). Personennahe Dienstleistungen in der Kinder- und Jugendhilfe zeichnen sich durch die Interaktion verschiedener Individuen innerhalb komplexer sozialer Prozesse aus. Im Zuge der immer weiter fortschreitenden Durchdringung des Alltags stellt sich daher die Frage, wie eine sinnvolle IT-Unterstützung personennaher Dienstleistungen gestaltet werden kann. Denn trotz der enormen Bedeutung der personenbezogenen Dienstleistungen in diesem Bereich existiert bisher nur eine fragmentarische Unterstützung der Arbeit durch dedizierte Informationssysteme. Die Probleme liegen dabei bei den einzelnen Systemklassen in folgenden Punkten.

Klassische Workflow-Systeme unterstützen lediglich klare starr formulierte Abläufe; der Flexibilität im Kontext personenzentrierter Dienstleistungen werden sie nicht gerecht. Kalendersysteme wie Outlook erlauben die individuelle Planung der Aufgabenerfüllung und die Koordination im Team. Es fehlt jedoch eine situationsbezogene Assistenz, welche die aktuelle Situation im Betreuungskontext berücksichtigt und eine zielorientierte Planung von Aktivitäten ermöglicht. Das Zusammenführen mehrerer Datenquellen und die Empfehlung geeigneter Aktivitäten unter Berücksichtigung langfristiger Ziele in der Dienstleistung können nicht unterstützt werden. Gemeinsamkeiten ähnlicher Zielstellungen und Kontexte können nicht in Form von Templates oder Empfehlungen berücksichtigt werden. Projektmanagementsysteme erlauben eine individuelle, einzelfallbezogene Arbeitsplanung. Ist solch ein System auf agile Praktiken ausgerichtet, ist auch eine hohe Flexibilität möglich. Ziele lassen sich bei der Planung berücksichtigen. Einige Nachteile der persönlichen Kalender sind so ausgeglichen. Eine Bestimmung der aktuellen Situation in der Dienstleistungserbringung ist hier jedoch nur eingeschränkt möglich, da die Aufgabensteuerung nicht so feingranular wie bei den vorbenannten Systemen erfolgt. Eine Integration von Assistenz und Datenfusion wären auch hier erst nachträglich möglich. Wie langfristige Ziele und kurzfristige Planung miteinander koordiniert werden können, müsste zusätzlich untersucht werden.

Bisherige branchenspezifische Informationssysteme unterstützen vor allem das Fallmanagement, die Dokumentation und Abrechnung sowie die Personaleinsatzplanung. Beispiele für derartige Systeme oder Anbieter sind UNION ALL GMBH, BEYOND SOFT GMBH (BEWO PLANER), DAARWIN BERATUNGSGESELLSCHAFT MBH, EDE DOKUMENTATIONSSYSTEM, ETHIS ${ }^{\circledR}$ (ETHIS ${ }^{\circledR}$ AMBULANT), FACTORIS, I-SOFT (LIFE PLUS), KILANKA, MEDIFOX und MICOS (MICOS KONZEPT) Sowie das modulare Werkzeug ENTERIO PAPP, um nur einige Beispiele zu nennen. Die Fähigkeiten derartiger Werkzeuge im Bereich der Prozesssteuerung und Assistenz sind allerdings noch weitgehend gering ausgeprägt. In besonderem Maße trifft dies auf proaktive Assistenzfunktionen zu. So konnten beispielsweise keine nennenswerten Empfehlungsfunktionen für den Vorschlag geeigneter Aktivitäten, Dokumente oder Ansprechpartner entdeckt werden.

Insgesamt besteht also noch erhebliches Potenzial für eine umfassende und maßgeschneiderte IT-Unterstützung im Bereich personennaher Dienstleistungen in der Kinder- und Jugendhilfe, vor allem im Bereich der Assistenz. Im Rahmen dieses Beitrags wird ein fortgeschrittenes Informationssystem konzipiert. Zunächst werden hierzu im Rahmen von Vorarbeiten bereits erhobene Anforderungen überblicksartig vorgestellt. Auf deren Grundlage wird anschließend die Gestaltung eines Informa- 
tionssystems vollzogen, das mit Hilfe von Nutzeroberflächenelementen beschrieben und anschließend im Hinblick auf die geschaffenen Potenziale von Praktikern bewertet wird. Anliegen der abgeleiteten Erkenntnisse ist es insgesamt, einen Beitrag zur Gestaltung innovativer IT-Systeme für personennahe Dienstleistungen im Bereich der Kinder- und Jugendhilfe zu leisten.

\section{Anforderungen zur IT-Unterstützung personenzentrierter Dienstleistungen}

Zur Entwicklung eines ganzheitlichen Systemmodells wurden zunächst Anforderungen ermittelt. Dazu wurden drei verschiedene Erhebungsinstrumente genutzt: Workshop, Experteninterviews und Shadowing. Ein solcher Einsatz unterschiedlicher Methoden hat den Vorteil, dass Unzulänglichkeiten einzelner Methoden oder singulärer Datenquellen kompensiert werden können (Oelerich und Otto 2011). Gelegentlich wird dies auch als Perspektiventriangulation bezeichnet (Flick 2011). Der entwickelte Anforderungskatalog wird detailliert von Lantow et al. (2020) beschrieben. Die Erhebung der Anforderungen hat über mehrere Runden in Workshops stattgefunden, an denen je mindestens 10 Personen aus drei unterschiedlichen Firmen teilgenommen haben. Die Workshop-Ergebnisse wurden durch sechs Tiefeninterviews ergänzt, die mit Experten unterschiedliche beruflicher Hintergründe (z.B. Pädagoge, Psychologe) geführt wurde. Weiter wurden ebenfalls sechs Beobachtungen der Arbeit durchgeführt (Shadowing) und die Beobachtungen wurden ebenfalls zur Ableitung von Anforderungen genutzt. Zur Strukturierung wurden die Leistungsphasen nach Fließ et al. (2015) herangezogen, diese sind Service, d. h. die eigentliche Leistungserbringung in Interaktion mit dem Kunden, Pre-Service und Post-Service, die die Vor- und Nachbereitung beschreiben. Personennahe Dienstleistungen sind jedoch oft in einen längerfristigen Prozess integriert, der längerfristige Ziele hat. Ein Beispiel wäre die Wiederherstellung der Mobilität im Rahmen der Physiotherapie. Dies bedeutet, dass Phasen der direkten, kurzfristigen Leistungserbringung mit übergreifenden Koordinationsaufgaben zur Zielerreichung verflochten sein können. Diese sind als eine zusätzliche Phase der Koordination zu erfassen. Aus der Sicht der Informationsverarbeitung ist auch die Einbeziehung eines Klienten wichtig; hier erfolgt die erste Information über den Fall. Diese Phase wird als Datenerfassung Klient bezeichnet. Neben diesen phasenspezifischen Anforderungen an die IT-Unterstützung für personenzentrierte Dienste gibt es auch allgemeine Anforderungen an entsprechende IT-Systeme im Kontext der personenzentrierten Dienstleistungen, die unter Allgemeine Anforderungen subsumiert werden. Tab. 1 zeigt den konsolidierten Anforderungskatalog.

In Bezug auf die Datenerfassung Klient müssen vielfältige Informationen aus verschiedenen Quellen berücksichtigt werden wie Information über die Person und von Seiten verschiedener Behörden wie dem Jugend- oder Sozialamt wie auch über das Familiensystem, die teils elektronisch, teils handschriftlich vorliegen. Das Familiensystem zeigt die wesentlichen Personen innerhalb eines Familienverbandes wie auch deren Relationen untereinander. Dabei können verwandtschaftliche, rechtliche (z.B. Erziehungsberechtigung) aber auch soziale Aspekte wie Loyalitäten, Macht- 
Tab. 1 Anforderungen zur IT-Unterstützung personennaher Dienstleistungen in der Kinder- und Jugendhilfe

Datenerfassung Klient - Anlegen des Falls im System

AN1.1 Berücksichtigung vielfältiger Informationsquellen

AN1.2 Anzeige freier Kapazitäten

AN1.3 Speicherung fallrelevanter Information

AN1.4 Definition von Fallverantwortlichen

AN1.5 Spezifikation von Zugriffsrechten auf das System (Rechtevergabe)

AN1.6 Modellierung von Familiensystemen

Pre-Service - Unterstützung der Vorbereitung von Aktivitäten wie Treffen mit dem Klienten

AN2.1 Informationsbereitstellung

AN2.2 Zentraler Zugriff zu Information

AN2.3 Informationsaustausch

AN2.4 Koordination der Akteure der konkreten Hilfeleistung

AN2.5 Visualisierung von Familiensystemen

AN2.6 Integration elektronischer Werkzeuge zur Informationsbeschaffung

Service - Verbesserung der Zusammenarbeit mit dem Klienten während des Hilfeprozesses

AN3.1 Visualisierung von Information

AN3.2 Zugriff auf einen zentralen, konsolidierten Informationsbestand (Single Point of Truth)

Post-Service - Erleichterung der Nachbereitung, z. B. Dokumentation der Tätigkeiten

AN4.1 Vereinfachte Dokumentation von Prozessen

AN4.2 Integration elektronischer Werkzeuge in die Informationsbeschaffung (z. B. Spracherkennung, GPS)

AN4.3 Erstellung von Rechnungen

AN4.4 Zielgruppenorientierter Informationsaustausch

AN4.5 Erfassung von Feedback

AN4.6 Terminmanagement

Weiterer Hilfeverlauf - Längerfristige Betrachtung und Koordination der Hilfe

AN5.1 Koordination der Akteure bezogen auf den gesamten Fall

AN5.2 Zielgruppenorientierter Informationsaustausch, fallbezogen

AN5.3 Zeitmanagement im Hilfeprozess

AN5.4 Aufgabenmanagement

AN5.5 Aufgabenstatus rückmelden

Allgemeine Anforderungen - Anforderungen an das System aus Sicht der Hilfeprozesse

AN6.1 Flexible Erweiterbarkeit

AN6.2 Situationsbezogene Unterstützung der Aufgabenausführung

AN6.3 Klienten-spezifische Aufgabensteuerung

AN6.4 Rollenspezifisches Aufgabenmanagement

AN6.5 Nutzerspezifisches Aufgabenmanagement

AN6.6 Empfehlungsfunktion für Aufgaben, Personen, Ressourcen

AN6.7 Sensor-Integration (z. B. GPS für aktuelle Position, Herzratenvariabilität zur Stresserkennung) 
positionen oder Koalitionen innerhalb der Familie festgehalten werden (Helming et al. 1999). Eine Sonderform bildet das Genogramm, das vor allem auf Verwandtschaftsverhältnisse fokussiert, jedoch auch weitergehende Information wie die Position in der Geschwisterreihe, welche die Eltern in der Herkunftsfamilie hatten, wie auch Todesfälle, Krankheiten und Symptome (Simon et al. 1999). Einem Klient muss mindestens ein zuständiger Mitarbeiter als Fallverantwortlicher sowie weitere Mitarbeitende als Fallbeteiligte zugewiesen werden, wozu die Kapazitäten der Mitarbeitenden ermittelt werden müssen. Weiter muss das Familiensystem modelliert werden können, um Zusammenhänge zwischen Personen anhand eines grafischen Schaubildes leicht erkennen zu können. Die gesammelte Information muss dauerhaft gespeichert werden können und zum Management der Information müssen Zugriffsrechte der Beteiligten definiert werden können.

In der Phase Pre-Service, also der Vorbereitung einer Hilfeleistung, ist eine gebündelte Informationsbereitstellung von großer Bedeutung, wobei der Zugriff auf eine zentrale Datenbasis erfolgen sollte, damit die Information stets aktuell ist. Zur Anbahnung der Hilfeleistung ist ein leichter Informationsaustausch zwischen allen beteiligten Akteuren wichtig. Die Fallbeteiligten sollten sich darüber hinaus über das System koordinieren können, d.h. die Planung, wer wann etwas wie durchführt. Zur Information über den Klient können die Fallbeteiligten auch das Familiensystem betrachten oder über fachspezifische Medien in Form von eBooks direkt im System anzeigen. Während der Erbringung der Hilfe, also in der Phase des Service, ist lediglich ein Abruf und eine Darstellung von Information wichtig, wobei auch auf einen zentralen, konsolidierten Informationsbestand zugegriffen werden sollte.

Die Phase Post-Service, also der Nachbereitung einer Hilfeleistung, ist vor allem die Vereinfachung der erforderlichen Dokumentation eine wesentliche Anforderung. Auch hier ist wieder die Integration elektronischer Werkzeuge relevant, z. B. der Einsatz von Spracherkennung oder die Erleichterung von Adresserfassungen über eine standortbasierte Vorschlagsfunktion. Auf Basis der gesammelten Information sollten Rechnungen automatisch generiert werden können und ein zielgruppenorientierter Informationsaustausch erleichtert werden. Mitarbeiter, die eine Rolle ausführen, könnten auf diese Weise gleich beim Vorliegen neuer Information benachrichtigt werden, also praktisch einem Fall „folgen“ bzw. Neuigkeiten abonnieren. Das System sollte weiter die Erfassung von Feedback während der Dokumentation erlauben, womit die Einschätzungen von Sozialarbeitenden im Hinblick auf erreichte Ziele mit entsprechender Begründung dokumentiert werden können. Die Nachbereitung umfasst zusätzlich auch Aspekte der Terminplanung, da anhand des Erreichten neue Aktivitäten geplant werden müssen. Über eine Verknüpfung mit einer Terminplanungskomponente oder Kalender sollten anhand dieser Parameter systemgenerierte Terminvorschläge generiert werden können.

Über die Planung, Durchführung und Nachbereitung konkreter Hilfeleistungen hinaus müssen längerfristige, fallbezogene Aspekte geplant werden können, wozu Anforderungen im weiteren Hilfeverlauf zu berücksichtigen sind. Diese bestehen in einer Koordination und dem zielgruppenspezifischen Austausch relevanter Information aller am Fall Beteiligten sowie in einer zeitlichen Planung des weiteren Hilfeprozesses insgesamt, d.h. längerfristig und über einzelne Hilfeleistungen hinausgehend. Das System sollte es dazu dem Anwender ermöglichen, im Rahmen eines 
Aufgabenmanagements verschiedenen fallbeteiligten Personen innerhalb eines Falls Aufgaben zuzuordnen und deren Bearbeitungstand zu verfolgen.

Über die bisher erläuterten Anforderungen hinaus ergeben sich schließlich weitere, allgemeine Anforderungen in Bezug auf die Systemgestaltung. Neben einer flexiblen Erweiterbarkeit betreffen diese Anforderungen vor allem eine der jeweiligen Situation und dem jeweiligen Nutzer angepasste Aufgabensteuerung, wobei unter „Nutzer" sowohl verallgemeinerte Rollen als auch konkrete Personen wie Mitarbeitende oder Klienten zusammengefasst werden. Durch die Umsetzung einer Empfehlungsfunktion für Aufgaben, Personen und Ressourcen kann der Aufwand zur Nutzung des Systems bedeutend gesenkt werden und der Komfort erhöht werden. Wird beispielsweise für einen Fall eine Aufgabe geplant, so könnte das System gleich Mitarbeitende vorschlagen, die kompetent für eine Aufgabe sind und zum gewählten Datum auch verfügbar sind.

\section{Entwurf eines Informationssystems}

In diesem Kapitel wird ein Systemmodell einer Anwendung vorgestellt, das basierend auf den zuvor genannten Anforderungen entwickelt wurde. In die Entwurfsaktivität flossen zusätzlich die aus den Interviews und Gesprächen vor Ort im Rahmen der Anforderungsanalyse gesammelten Einblicke mit ein. In diesem Sinne wurden die Entwurfsaktivitäten nicht streng auf die Erfüllung der Anforderungen reduziert. Vielmehr dienten die Anforderungen als Grundgerüst, um den Systemvorschlag aufzubauen. Für die Veranschaulichung des Systemmodells werden eine Navigationsstruktur und Mockups präsentiert. Die Navigationsstruktur dient der Orientierung innerhalb der verschiedenen Anwendungsfenster und -ebenen des Systems und ermöglicht die Veranschaulichung von Verknüpfungen innerhalb der verschiedenen Arbeitsbereiche. Mockups demonstrieren die Struktur sowie die geplanten Funktionen des Systems und besitzen gegenüber Prototypen den Vorteil, dass der Endanwender bereits zu einem frühen Entwicklungszeitpunkt einen Eindruck gewinnen kann. Sie werden generell für Präsentationszwecke eingesetzt (vgl. Schickler et al. 2015). Eine Präsentation ist notwendig, um den Entwurf einer interviewgestützten Evaluation mit Praktikern unterziehen zu können (vgl. Abschn. 4).

\subsection{Navigationsstruktur des Systems}

Die Navigationsstruktur ist in Abb. 1 veranschaulicht. Sie zeigt die verschiedenen Arbeitsbereiche und ihre jeweiligen Fenster und ist in verschiedene Ebenen gegliedert, wobei methodisch Zühlke (2011) gefolgt wird.

Auf der Ebene 1 befindet sich die Startseite. Diese stellt eine übersichtartige Ansicht aktueller Termine, Fälle und Aufgaben in reduzierter Form zur Verfügung. Auf der Ebene 2 sind die bereits auf der Startseite in vereinfachter Form dargestellten drei Hauptarbeitsbereiche in ihrem vollen Funktionsumfang verfügbar und ihre Verknüpfungen sind ersichtlich. Der Arbeitsbereich der Fälle untergliedert sich weiter in Ebene 3. Diese umfasst die Fallbearbeitung, eine Assistenzfunktion (wird 


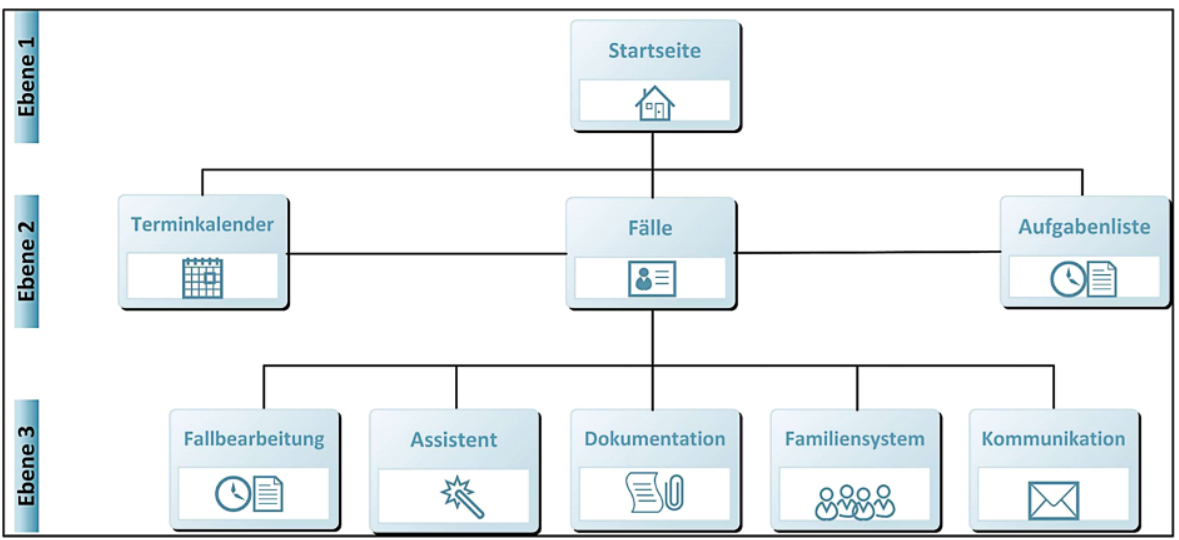

Abb. 1 Navigationsstruktur des Systems

im folgenden Abschnitt weiter erläutert), die Dokumentation, das Familiensystem sowie die Kommunikation.

\subsection{Entwurf der Arbeitsbereiche als Mockup}

Für die Visualisierung der zuvor systematisierten Arbeitsbereiche mithilfe der Navigationsstruktur wurden Mockups angefertigt. Relevant für weitere Erklärungen sind die drei Hauptarbeitsbereiche der Ebene 2 und ausgewählte Arbeitsbereiche der Ebene 3, die auch später im Rahmen der Interview-basierten Evaluation einer kritischen Beurteilung durch Fachexperten unterzogen wurden.

Terminkalender Unterstützt die Phase des Pre-Service, Service und die Planung des weiteren Hilfeverlaufs. Konkrete, unterstützte Anforderungen in diesem Bereich sind die in Tab. 1 eingeführten Anforderungen (im Folgenden kurz als „AN“ bezeichnet, gefolgt von der Nummer aus Tab. 1) AN 2.1-2.4, 3.1-3.2, 4.3, 4.6, 5.1 und 5.3. Der Terminkalender sollte neben den bereits eingetragenen Terminen auch die dem Systemnutzer zugeordneten Fälle zum Zugriff anbieten. Dies ist für das Eintragen neuer Termine von Bedeutung (AN2.4). Weiter sollte der Kalender in einer Tages- und Wochensicht über beteiligte Personen, Anlass und Ort informieren (AN2.1, 2.2, 2.4, 3.1, 3.2, 5.1, 5.3). Jeder Terminkalender-Account sollte weiter mit den spezifischen Fällen des Anwenders verknüpft sein. Mit Hilfe dieser Verknüpfung kann das System nur die für den Nutzer relevanten Fälle für die Auswahl in der Terminerstellung vorschlagen (AN2.1, 2.2). Durch die Auswahl des Falls greift das System wiederum auf die im Fall hinterlegten Personen zurück, um diese für die Auswahl bereitzustellen. Alle beteiligten Personen werden bei Terminveränderungen automatisch durch das System informiert und können die Änderung akzeptieren oder zurückweisen (AN2.2). Die in der Auswahl vorgeschlagenen Orte werden auf Grundlage häufig verwendeter Orte im Zusammenhang mit dem Fall angeboten. Zu den einzelnen Terminen lassen sich Dokumente und Dateien anfügen (AN3.2). Als Alternative zum selbständigen Festlegen des Zeitpunktes, an dem der Termin stattfin- 
det, bietet das System die Funktion der Termingenerierung. Diese benötigt als Input den Fall, beteiligte Personen, den Ort und den gewünschten Zeitraum. Im Weiteren muss eine von drei Dimensionen (Zeit, Ort und Dringlichkeit) zur Planung priorisiert werden. Bei der Auswahl von Zeit verfolgt das System das Ziel, einen Termin zu finden, der möglichst dem vorher angegebenen Zeitfenster entspricht und Vor- und Nachbereitung des Termins berücksichtigt (AN4.6, 5.3). Wird Ort gewählt, werden Termine entsprechend ihrer geografischen Nähe angeboten. Bei Dringlichkeit wird der nächste freie Termin vorgeschlagen. Weiter werden alle intern an einem Fall beteiligten Personen durch eine Einladung informiert und um Rückmeldung gebeten (AN2.3, 4.6, 5.3).

Fallbearbeitung Die Fallbearbeitung beinhaltet alle mit dem Fall verbundenen Aktivitäten und Informationen und unterstützt damit die Phasen Datenerfassung Klient, Pre-Service, Service, Post-Service und Weiterer Hilfeverlauf (AN1.1, 1.3-1.6, 2.1-2.5, 3.1, 3.2, 4.1, 4.4, 4.5, 5.1, 5.2). Aktivitäten stellen Aufgaben dar, die der Anwender für den Fall definiert und mit einem Erledigungsdatum vermerkt. Das Anwendungsfenster in Abb. 2 zeigt überblicksartig alle untergeordneten Arbeitsbereiche der Fallbearbeitung (AN1.3, 1.4, 2.1, 2.2). Sie können entsprechend vergrößert werden, um in die eigentliche Bearbeitung der Aktivität zu gelangen.

Das obere linke Fenster zeigt die aktuelle Aktivität (vgl. Markierung 1). Der Anwender hat diese Aktivität im System hinterlegt und mit den entsprechenden fall- und

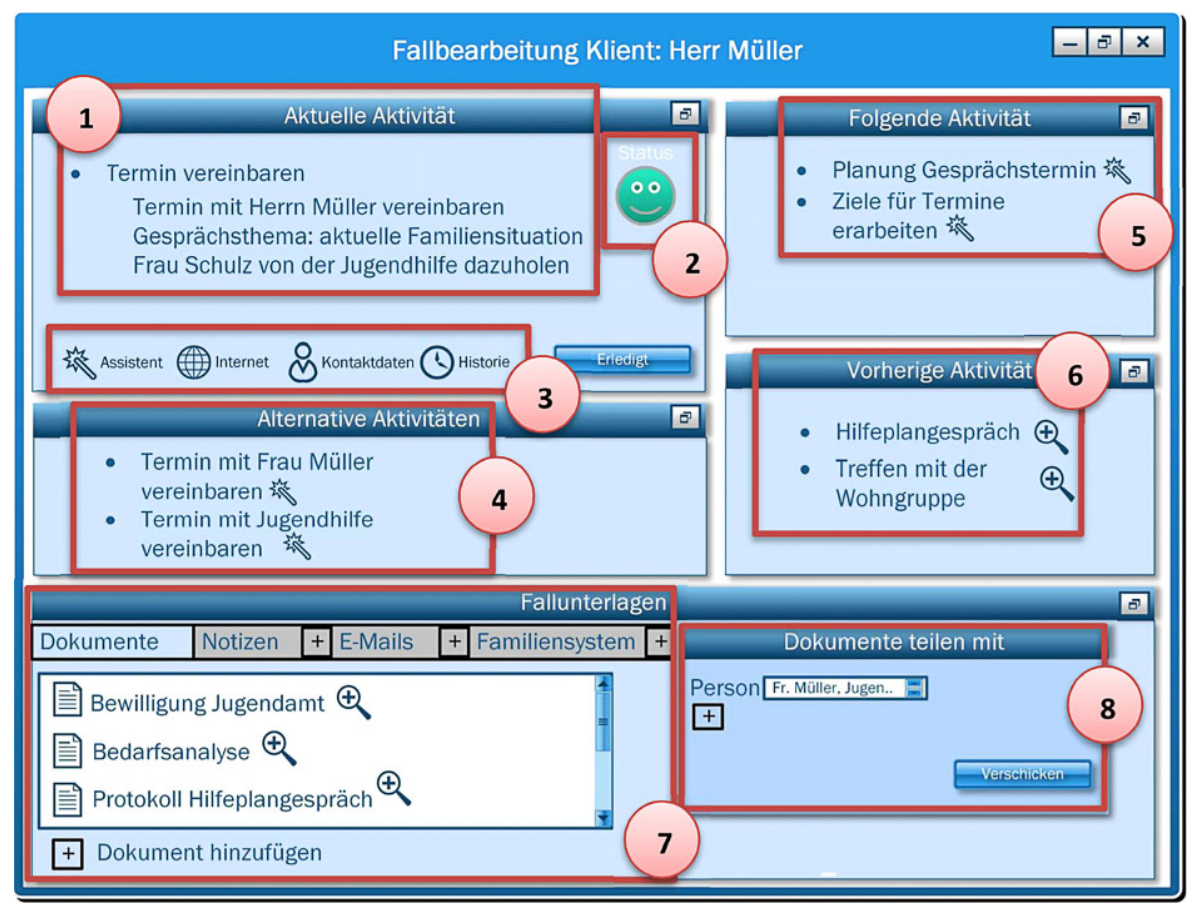

Abb. 2 Fallbearbeitung 
aktivitätsbezogenen Informationen ergänzt. Zusätzlich ordnet der Anwender die Aktivität einer Kategorie zu, z. B. die Vorbereitung auf einen Termin. Diese Einordnung ist für die spätere Erklärung der Assistenzfunktionen von Bedeutung (vgl. Markierung 3, AN6.6). Über den Bearbeitungsstand der Aufgabe informiert die Anzeige (vgl. Markierung 2). Diese ist als eine Art Ampel aufgebaut und signalisiert den Handlungsbedarf hinsichtlich der zu erledigenden Aktivität. Den Status der Anzeige generiert das System durch den Abgleich vom geplanten Beendigungszeitpunkt und dem aktuellen Datum mit dem jeweiligen Bearbeitungsstand der Aktivität. Die Aktivitäten der Fallbearbeitung und der Aufgabenliste (vgl. auch Abb. 4) werden miteinander synchronisiert. Zur Unterstützung der zu leistenden Aktivitäten sind die darunterliegenden Bedienelemente vorgesehen (vgl. Markierung 3). Der Assistent stellt zusätzliche Funktionen des Systems in Abhängigkeit der Aktivitätskategorie zur Verfügung. Bei vorbereitenden Aktivitäten bietet der Assistent Informationen zu den Interessen des Klienten an, um die Suche nach einer möglichen Aktivität für den Termin zu erleichtern (AN6.6). Handelt es sich um Aktivitäten wie die Durchführung des Termins mit dem Klienten, könnte der Assistent das Verschicken von festgelegten Gesprächszielen auf ein mobiles Endgerät veranlassen, um diese dem Anwender nochmals zu visualisieren. Neben den Assistenzfunktionen in Markierung 3 stellt das System auch den Schnellzugriff auf das Internet für die Informationsbeschaffung zur Verfügung, wobei relevante Funde dem entsprechenden Fall in der Dokumentation (vgl. Markierung 7) angehängt werden können. Über das Bedienelement der Kontaktdaten werden alle mit dem Fall verknüpften Personen angezeigt, welche direkt kontaktierbar sind. Die Historie enthält die detaillierte Ausgabe aller bis dahin erfolgten Aktivitäten und beteiligten Personen des Falls in einem Zeitstrahl. Somit lässt sich der gesamte Fallverlauf nachvollziehen (AN1.1, 1.3, 2.2, 3.2).

Als eine weitere Assistenzfunktion stellt das System selbständig Alternativen (vgl. Markierung 4) zur aktuellen Aktivität bereit. Diese resultieren aus vergangenen Hilfeverläufen von Systemanwendern, die ähnliche Fälle bearbeitet haben. Das Vorschlagen Folgeaktivität (vgl. Markierung 5) stellt eine zusätzliche Assistenzfunktion dar (AN6.6). Die vorherige Aktivität (vgl. Markierung 6) ermöglicht die Rückkehr in die unmittelbar zuvor durchgeführte Aktivität. Der Anwender kann Änderungen an bereits abgeschlossenen Aktivitäten vornehmen, beispielsweise durch Ergänzen von Notizen oder Dokumenten. Diese sind zur besseren Nachvollziehbarkeit als „nachträglich ergänzt“" gekennzeichnet, z. B. im Fallverlauf (vgl. Markierung 3). Alternativ informiert sich der Anwender über die zuvor stattgefundene Aktivität. Zur Verwaltung der Fallunterlagen dient die Bedienoberfläche im unteren Teil des Anwendungsfensters (vgl. Markierung 7). Sie umfassen Dokumente, Notizen, E-Mails und das Familiensystem (AN2.5). Der Anwender kann die Fallunterlagen ergänzen und mit Aktivitäten verknüpfen. Fallunterlagen können per E-Mail mit fallrelevanten Personen über ein Bedienelement (vgl. Markierung 8, AN2.3) geteilt werden.

Zur Ansicht einer grafischen Darstellung des Familiensystems des Klienten in Form eines Genogramms gelangt der Anwender durch Auswahl des Reiters Familiensystem (vgl. Abb. 3) (AN2.5). Ein Genogramm dient der Veranschaulichung von Familienkonstellationen bzw. des sozialen Umfeldes des Klienten (vgl. Simon et al. 1999). Infolgedessen ist es für den Anwender einfacher, Wirkungszusammenhänge zu erkennen, welche von Relevanz für die Fallbearbeitung sind. Durch das 


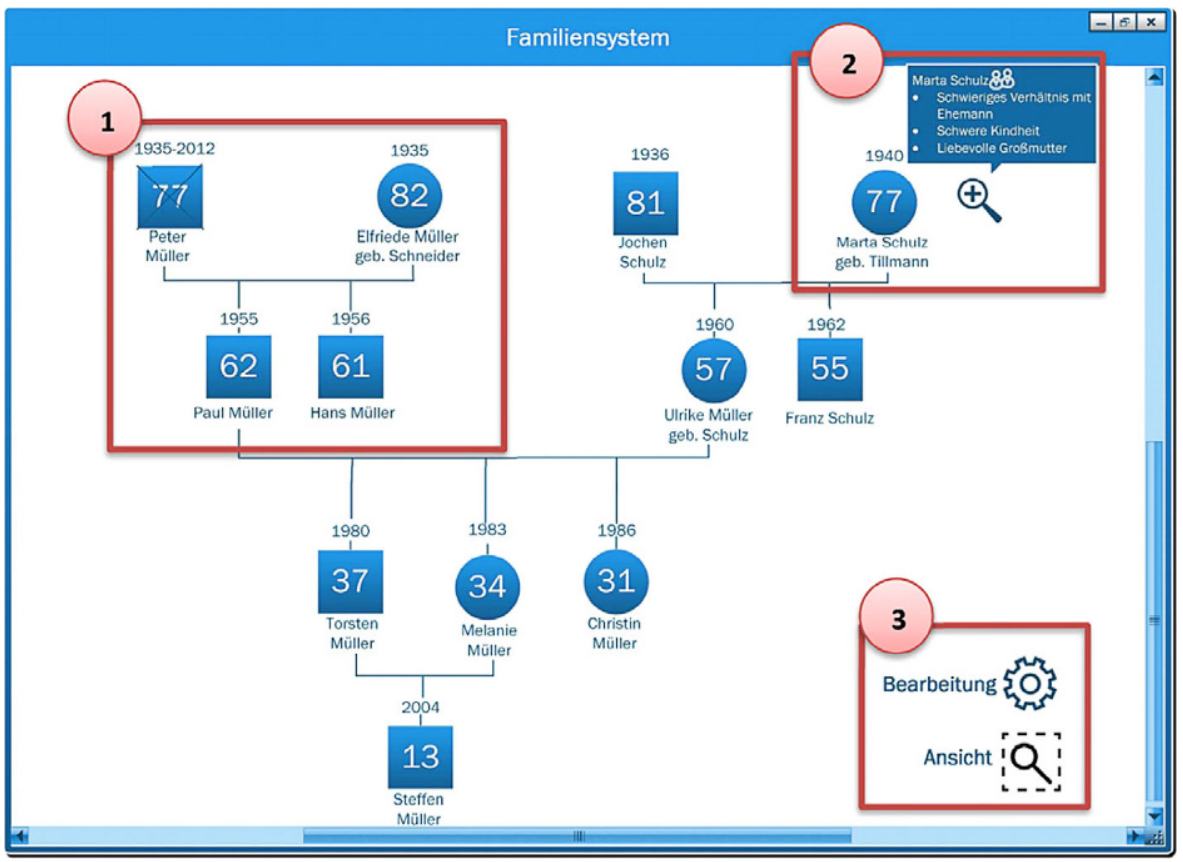

Abb. 3 Beispielhafte Darstellung eines Genogramms

Markieren einzelner Personen, erhält der Anwender zusätzlich Informationen (vgl. Markierung 1, 2) zu der Person. Für eine detaillierte Ansicht wird über das entsprechende Bedienelement (vgl. Markierung 3) der dargestellte Detailgrad verändert.

Aufgabenliste Die Aufgabenliste (vgl. Abb. 4) enthält die fallübergreifende Ansicht aller Aktivitäten und unterstützt vornehmlich die Phase der Planung, d.h.

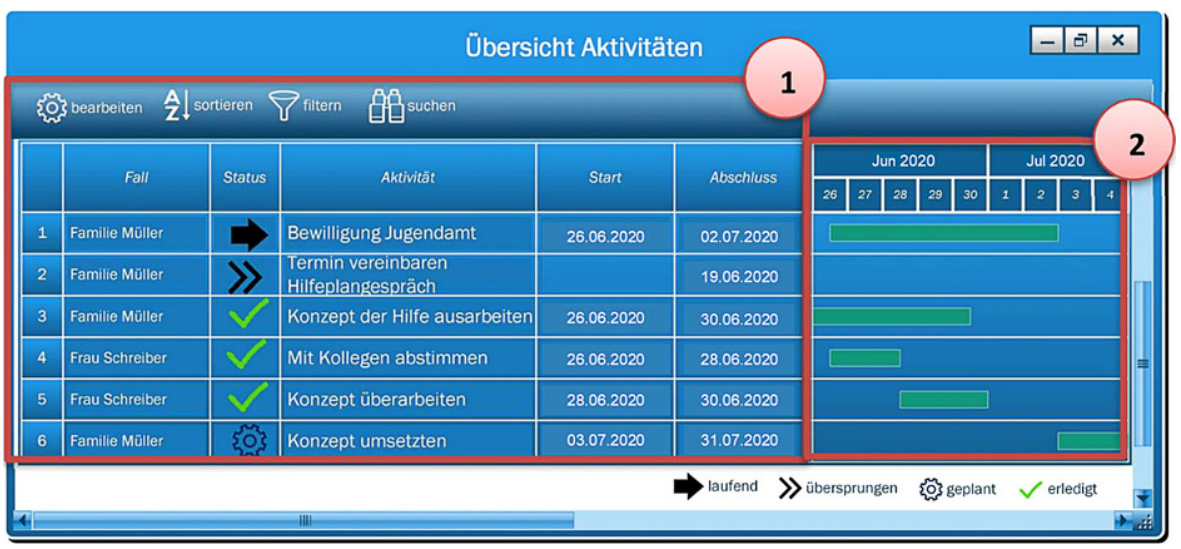

Abb. 4 Aufgabenliste 
Pre-Service, sowie eine längerfristige Betrachtung und Koordination der Hilfe (AN2.1-2.3, 5.1, 5.3-5.5). Dort sind alle zu erledigenden Aktivitäten (vgl. Markierung 1) mit Bearbeitungsstatus und dem zugehörigen Fall aufgeführt. Die Aufgabenliste ermöglicht dem Anwender die notwendige Übersicht über seine Fälle (AN5.4). Er hat weiter die Möglichkeit, die Aktivitäten innerhalb der Liste zu bearbeiten und neue Aktivitäten hinzuzufügen. Dort getätigte Veränderungen und der aktuelle Status werden automatisch in die Fallbearbeitung (vgl. Abb. 2) übernommen (AN2.2). Aktivitäten können darüber hinaus sortiert, gefiltert und durchsucht werden. Für den in Abb. 4, Markierung 2 ersichtlichen Status besitzt jede der Aktivitäten einen Startund Endtermin, welcher rechts im Anwendungsfenster veranschaulicht ist (AN5.5).

Die allgemeinen Anforderungen AN6.1-6.7 sind keiner spezifischen Komponente zuzuordnen, da sie vom Charakter her querschnittig sind. So betrifft z. B. die flexible Erweiterbarkeit (AN6.1) alle Komponenten des Systems.

\section{Interview-basierte Evaluation}

Für die Bewertung von IT-Systemen sind in der Literatur zahlreiche Kriterien und Modelle zu finden, allerdings oft für die Bewertung bereits im Einsatz befindlicher Systeme. Bei dem im vorigen Kapitel vorgestellten Systemmodell handelt es sich jedoch um einen Systementwurf. Für die Evaluierung des Systems muss in der Folge eine Ex-ante-Bewertung vor der Implementierung vorgenommen werden, wie dies auch von Sonnenberg und v. Brocke (2012) im Entwicklungsprozess von Artefakten vorgeschlagen wird. Eine geeignete Methode hierzu bilden unter anderem Experteninterviews nach Sonnenberg und v. Brocke (2012).

Für die Bewertung des Systemmodells wurden dieselben Experten herangezogen, die bereits zur Erhebung der Anforderungen befragt und beobachtet wurden. Sie sind auch die beabsichtigten späteren Nutzer des Systems. Bei den ausgewählten Experten handelt es sich zum überwiegenden Teil um Sozialarbeiter, die in der Jugendhilfe tätig sind. Unter den befragten Expertinnen und Experten sind zwei Frauen und fünf Männer. Die Befragten weisen als Sozial-, Medien- und Sonderpädagogen sowie Sozialarbeiter ein breites Tätigkeitsspektrum auf, wobei der Median der Beschäftigungsdauer bei 10 Jahren liegt. Darüber hinaus ist ihr beruflicher Alltag sowohl durch den Kontakt mit dem Klienten, als auch von organisatorischen und administrativen Tätigkeiten geprägt. Als ein weiterer Experte wurde ein Systemadministrator mit detaillierten Kenntnissen über den Anwendungskontext des Systems befragt. Zur Durchführung der Experteninterviews wurde ein Interviewleitfaden entwickelt. Die für den Einstieg in das Gespräch verwendeten Probleme des Arbeitsprozesses wurden den zuvor geführten Experteninterviews entnommen und besitzen einen realistischen Bezug zum Anwendungskontext des Systems. Weitere Fragen bezogen sich auf die Informationsbereitstellung, wobei auch auf den Begriff der Informationsqualität nach DeLone und McLean (1992) Bezug genommen wurde. Weitere Fragen bezogen sich auf den Nutzen der Information. Konkret beinhaltete dies Fragen $\mathrm{zu}$ einer möglichen Zeitersparnis, des Informationsstands bei Entscheidungen und der Effizienz bei Arbeitsprozessen. Ergänzt wurde der Nutzen mit der Einschätzung hinsichtlich der verschiedenen Phasen des Dienstleistungsprozesses nach Fließ 
et al. (2015). Abschließend wurde nach der Absicht zur Nutzung des Systems gefragt, die einem der zentralen Konstrukte des etablierten Technology Acceptance Model (TAM) von Davis (1985) entspricht. Im Folgenden werden die Ergebnisse der Experteninterviews präsentiert.

Nutzen des Systems Den Nutzen des Systems für die Arbeit bewerteten alle Experten einheitlich als gegeben. Worin sie dies begründet sahen, differenzierte sich teilweise innerhalb der befragten Experten. Die Befragten nannten vor allem durch das System bereitgestellte Funktionen, aus denen Nutzenaspekte für ihre Arbeit entstehen, z. B. das vereinfachte in Kontakt treten mit Personen und die sich anschließende Terminfindung. Als weiterer Punkt ist der Vernetzungsgedanke zwischen den Kollegen mit Informationen genannt worden und darüber hinaus die generell erleichterte Informationsbeschaffung. Der überwiegende Teil der Experten bewertete dabei den Umfang der bereitgestellten Informationen nach Vorstellung des Systementwurfs als optimal. Die Menge der dargestellten Information sollte nicht erhöht werden, damit laut Aussage der Experten die „Alltagspraktikabilität“ nicht verloren ginge. Für einen kleinen Teil der Interviewpartner sind bereits die jetzt dargestellten Informationen zu viel. Ebenfalls als nützlich angeführt wurden die in der Fallbearbeitung durch das System generierten Vorschläge für Aktivitäten.

Hinsichtlich einer möglichen Zeitersparnis, die durch ein solches System geschaffen werden kann, urteilten die Experten unterschiedlich. Der überwiegende Teil vertritt die Auffassung, dass Zeit eingespart werden kann. Unterschiede bei den Vertretern dieser Sichtweise gibt es hinsichtlich des Zeithorizontes, wann dieser Effekt eintritt. Hinsichtlich möglicher positiver Effekte für die Entscheidungsfindung urteilten die befragten Experten ebenfalls unterschiedlich. Der größte Teil vertritt die Auffassung, dass Entscheidungen durch die vom System bereitgestellten Informationen informierter getroffen werden können. Die Experten sehen dies vor allem in der schnelleren Verfügbarkeit von Informationen begründet.

Bei der Bewertung der durch das System geschaffenen Effizienz sind die Experten ebenfalls geteilter Meinung. Grundsätzlich stimmen alle Experten möglichen Effizienzsteigerungen zu. Unterschiede in den Positionen werden hingegen deutlich, wenn die tatsächliche Realisierung dieser Effizienzsteigerungen betrachtet wird. So sehen zwei der befragten Experten viele Hürden die verhindern, dass theoretisch begründete Effizienzsteigerungen auch in der Praxis realisierbar sind. Die Experten bezweifeln in diesem Zusammenhang die konsequente Nutzung des Systems aufgrund mangelnder Akzeptanz bei den vorgesehenen Anwendern. Für die Mehrheit der Experten sind die Effizienzsteigerungen jedoch erzielbar. Den Grund für die Effizienzsteigerungen sehen sie vor allem in einem besseren Wissensmanagement und einfacherem Zugang zu benötigter Information. In diese Richtung deuten auch Aussagen zur Struktur des Systems. So wurde durch den überwiegenden Teil der Experten die durch das System geschaffene Struktur als zentral bezüglich des Nutzens wahrgenommen. Die Experten empfanden die Struktur als logisch und nachvollziehbar. Weiter sehen als eine wesentliche Voraussetzung für den Nutzen des Systems einige der Experten die konsequente Nutzung durch alle Anwender an. 
Auftreten des Nutzens entlang der Phasen des Hilfsprozesses Die Experten sehen grundsätzlich in allen Phasen des Dienstleistungsprozesses einen Nutzen durch das System. Dieser liegt laut Experten vor allem darin begründet, dass die Informationen, die das System zur Verfügung stellt, nicht nur relevant für die Vor- und Nachbereitung der Dienstleistung sind, sondern auch eine Bedeutung für den Kontakt mit dem Klienten besitzen. Für einen der Experten knüpft sich dies jedoch an die Bedingung, dass der betrachtete Kontakt mit dem Klienten im Vorfeld ausreichend antizipierbar ist. Das System ist darauf angewiesen, dass entsprechende Information zu dem bevorstehenden Kontakt eingepflegt werden. Wenn dies jedoch nicht gewährleistet ist, kann das System die Komplexität der Kontaktsituation mit dem Klienten nicht abbilden und in der Folge nicht durch die Bereitstellung entsprechender Informationen unterstützen. Daraus resultieren unter anderem auch der einfachere Zugang zu Informationen und die zentrale Speicherung der Information an einem Ort. Ein weiterer Aspekt, der genannt wurde betraf den Sachverhalt, dass sämtliche Arbeitsbereiche in einem System vereint sind. Das Vernetzten der verschiedenen professionellen Akteure miteinander aber auch die Einbindung der Klienten in das System und der darüber ermöglichte Informationsaustausch wird ebenfalls als Bereicherung empfunden. Die damit verbundene gestiegene Transparenz in den Arbeitsprozessen ist ein weiterer Nutzenaspekt, der genannt wurde. Die Experten erwähnen weiterhin den gestiegenen Komfort innerhalb der Arbeit für die Mitarbeiter, die mit Hilfe des Systems zeitlich flexibler einzelne Arbeitsprozesse erledigen können. Ein anderer Aspekt des Nutzens entstand aus der verbesserten terminlichen Verwaltung. Auf Ebene des Managements wurde durch die Experten die vereinfachte Schaffung eines Überblicks über relevante Zahlen für die Geschäftsleitung angeführt.

Absicht zur Nutzung des Systems Die Frage nach der Absicht der Nutzung des vorgestellten Systems beantworteten alle Experten geschlossen. Jeder der Befragten äußerte seine grundsätzliche Bereitschaft zur Nutzung des Systems. Ob das System dann auch weiterhin Verwendung findet, knüpften die Experten an die Bedingung, dass sich das System in der Praxis als sinnvoll herausstellt. Nur einer der befragten Experten konnte eine vollständige Nutzung des Systems mit allen zur Verfügung gestellten Funktionen bereits zum jetzigen Zeitpunkt ausschließen. Diese kritische Selbsteinschätzung erfolgte mit der Begründung, dass nicht die notwendige Bereitschaft vorhanden sei, die Informationen im benötigten Detailgrad im System einzupflegen. Andererseits äußert die identische Person ihre Bereitschaft zur Nutzung aller Systemfunktionen, welche die Zusammenarbeit mit den Kollegen umfasst, denn dort erkennt der Experte den größten Nutzen für sich. Dies deutet auf die Wichtigkeit eines wahrgenommenen Nutzens bezüglich der Nutzungsabsicht hin, die auch im Modell von DeLone und McLean (1992) betont wird. Alle befragten Experten bestätigten weiter, dass die empfundenen Schwierigkeiten in ihren Arbeitsprozessen sie zu einer Systemnutzung animieren würden.

Hürden zur Nutzung des Systems Als Hürden wurden zahlreiche Aspekte genannt, die sich in verschiedene Kategorien einteilen lassen. Eine besonders häufig genannte Hürde stellt der Faktor Mensch dar. Darunter fallen Aspekte wie die Be- 
reitschaft, sich auf neue Instrumente bei der Arbeit einzulassen, das Ablegen von bisherigen Gewohnheiten in den Arbeitsprozessen oder die Skepsis neuen Hilfsmitteln gegenüber. Aber auch das technische Verständnis kann eine Hürde sein. Eine weitere Kategorie sind die geschaffenen organisationalen bzw. gesetzlichen Rahmenbedingungen. Ein Experte erklärte, dass die durch das System erforderlichen Dokumentationen in ihrem vorgesehenen Umfang nicht vollständig abrechenbar wären. Als eine weitere Hürde wird aus Sicht der Experten der generelle Mehraufwand betrachtet, der durch die Nutzung des Systems entsteht, sofern viele Dokumentationen doppelt erfolgen, $d$. h. sowohl analog als auch digital. Diese Probleme betreffen viele wissensorientierte Systeme, sodass auch Lösungen aus diesem Bereich prinzipiell einsetzbar scheinen und im Rahmen zukünftiger Forschung zu untersuchen sind. Darüber hinaus nannten die Experten als Hürde die nicht unmittelbare Verfügbarkeit des Systems im Vergleich zu Stift und Papier. Ergänzend erwähnt wird hierbei der wiederholte Wunsch nach einer Anwendung auf dem Smartphone als Erweiterung des eigentlichen Systems.

Risiko bei der Nutzung des Systems Da bisher im Wesentlichen Chancen und Nutzen aus Expertensicht erfragt wurden, sollte im Weiteren auch das Risiko eingeschätzt werden. So könnte ein mögliches Risiko der Verlust zwischenmenschlicher Kontakt sein. Die Experten vertraten übereinstimmend die Auffassung, dass sie darin keine Gefahr sehen. Vielmehr stellt das System durch die bereitgestellten Informationen oftmals eine Anregung bzw. Erinnerung zur Kontaktaufnahme dar. Das System unterstützt den sozialen Kontakt, substituiert ihn jedoch nicht. Inwieweit der Datenschutz eine Hürde für die Systemnutzung darstellt, wird kontrovers unter den Experten betrachtet. Der überwiegende Teil der befragten Experten sieht in der Einhaltung des Datenschutzes durch die Nutzung des Systems keine Schwierigkeiten. Aus der Perspektive der Experten stellt das Verwalten der persönlichen Daten im System kein erhöhtes Risiko dar, da ähnliche Systeme in der Praxis bereits Anwendung finden, ohne dass es zu Verletzungen des Datenschutzes kam bzw. unter Einhaltung relevanter Richtlinien und Gesetze wie der DSGVO.

\section{Fazit und Ausblick}

Da bisher kaum eine adäquate Unterstützung der Arbeit durch dedizierte Informationssysteme existiert, wurde im vorliegenden Beitrag der Versuch unternommen, Strukturen für ein umfassendes branchenspezifisches Informationssystem zur Unterstützung personennaher Dienstleistungen im Bereich der Kinder- und Jugendhilfe zu entwerfen. Hierzu wurde die Gestaltung eines Informationssystems vorgenommen, das hinsichtlich seiner Navigationsstruktur und seiner Komponenten über Mockups beschrieben wurde. Die Generalisierungsreichweite des Systemvorschlags umfasst all jene Szenarien, in denen personennahe Dienstleistungen erbracht werden, die (i) Gespräche und therapeutischen Interaktionen mit den Klienten über einen längeren Zeitraum umfassen, (ii) eine individuelle Absprache der Termine und Maßnahmen zwischen einem oder mehreren Klienten und einem oder mehreren Hilfspersonen erfordern und (iii) eine Ergebnisdokumentation erfordern. Der Systemvorschlag 
ist also für eine große Anzahl an Jugendhilfe-Einrichtungen und Jugendzentren relevant.

Der Systemvorschlag wurde im Hinblick auf die geschaffenen Potenziale von Praktikern bewertet. Neben einzelnen Nutzenkomponenten wie der Einsparung von Zeit oder der Unterstützung von Entscheidungen durch die passgenaue Informationsbereitstellung wurde überraschenderweise vor allem in der Strukturierung der Domäne und der nahtlosen Verknüpfung unterschiedlicher Informationsarten wie Terminkalender, Fallbearbeitung und Aufgabenliste mit ihren jeweiligen Teilinformationen zu Aktivitäten und Fällen ein großer Mehrwert gesehen. Dies zeigt, dass Informationssysteme letztendlich nicht nur von vornerein feststehende Bedarfe erfüllen, sondern strukturierend auf die realweltlichen Phänomene der Arbeitswelt einwirken. Der überwiegende Teil der Experten bewertete dabei den Umfang der bereitgestellten Informationen nach Vorstellung des Systementwurfs als adäquat.

Hinsichtlich der Nutzung eines derartigen Systems haben alle befragen Praktiker ihre grundsätzliche Bereitschaft und ihr Interesse an der Nutzung des Systems geäußert. Diese Motivation speist sich vor allem aus den wahrgenommenen Herausforderungen der Kommunikations- und Informationsprozesse im Arbeitsalltag. Zusammenfassend können daher aus der bisherigen Entwicklung des Systementwurfs und seiner Evaluation mit Experten folgende Erkenntnisse festgehalten werden:

- Die befragten Experten haben der Strukturierung der Domäne durch IT und der nahtlosen Verknüpfung unterschiedlicher Komponenten wie Terminkalender, Fallbearbeitung und Aufgabenliste eine hohe Bedeutung beigemessen. Hieraus lässt sich ableiten, dass ein Informationssystem vor allem gut integriert sein sollte und relevante Vorlagen für Dokumente und Aktivitäten beinhalten und proaktiv anbieten sollte.

- Hinsichtlich der Zeitersparnis war das Meinungsbild der Experten durchwachsen. Daher muss darauf geachtet werden, dass die Komplexität des Systems auf das unbedingt erforderliche Maß reduziert wird, um Einarbeitungs- und Lernaufwände zu reduzieren. Weiter muss dafür Sorge getragen werden, dass keine Doppelarbeit entsteht, etwa weil Information papierbasiert erfasst und parallel im System gepflegt wird.

- Hinsichtlich des Datenschutzes und auch des möglichen Risikos, dass die menschliche Interaktionsqualität leidet, wurden kaum Bedenken geäußert. Das Informationssystem sollte in jedem Falle konform zu geltenden Gesetzen im Bereich des Datenschutzes und der Datensicherheit entwickelt werden und relevante ethische Standards und Richtlinien sollten eingehalten werden.

Insgesamt zeigt sich, dass branchenspezifische Informationssysteme für die Kinder- und Jugendhilfe über ein hohes, noch nicht annähernd ausgeschöpftes Potenzial zur Unterstützung von Arbeitsprozessen verfügen. Wir hoffen, mit unserem Beitrag einen Bezugsrahmen und eine Inspirationsquelle für die Entwicklung zukünftiger Systeme zu liefern, insbesondere, was die Komponenten solcher Systeme und ihre Bezüge untereinander anbelangt.

Im Rahmen der zukünftigen Forschung werden wir weiter untersuchen, wie Verfahren aus dem Bereich der Daten- und Textanalyse in ein derartiges System integriert werden können, um noch weitergehende Funktionalitäten vor allem im Bereich 
der Analyse und Prognose von Fällen bereitstellen zu können. Ein weiteres Feld zukünftiger Forschung bildet Integration von Sensorik, etwa zur Stresserkennung, um über diesbezügliche Reflexionsprozesse schließlich die Gesundheit und das Wohlergehen der Nutzer zu steigern. Eine permanente Evaluation und Rückkoppelung der Entwicklungsaktivitäten mit den Nutzern erscheint in diesem Zusammenhang zukünftig wichtig. Ergänzend kann eine permanente empirische Begleitforschung und Evaluation etwa in Form von Tiefeninterviews oder Experimenten zum Vergleich traditioneller Werkzeuge mit dem spezifisch entwickelten Werkzeug wichtige Erkenntnisse zum Wert und Beitrag der IT liefern.

Funding Open Access funding provided by Projekt DEAL.

Open Access Dieser Artikel wird unter der Creative Commons Namensnennung 4.0 International Lizenz veröffentlicht, welche die Nutzung, Vervielfältigung, Bearbeitung, Verbreitung und Wiedergabe in jeglichem Medium und Format erlaubt, sofern Sie den/die ursprünglichen Autor(en) und die Quelle ordnungsgemäß nennen, einen Link zur Creative Commons Lizenz beifügen und angeben, ob Änderungen vorgenommen wurden.

Die in diesem Artikel enthaltenen Bilder und sonstiges Drittmaterial unterliegen ebenfalls der genannten Creative Commons Lizenz, sofern sich aus der Abbildungslegende nichts anderes ergibt. Sofern das betreffende Material nicht unter der genannten Creative Commons Lizenz steht und die betreffende Handlung nicht nach gesetzlichen Vorschriften erlaubt ist, ist für die oben aufgeführten Weiterverwendungen des Materials die Einwilligung des jeweiligen Rechteinhabers einzuholen.

Weitere Details zur Lizenz entnehmen Sie bitte der Lizenzinformation auf http://creativecommons.org/ licenses/by/4.0/deed.de.

\section{Literatur}

Bsirske F (2014) Digitalisierung und Dienstleistungen - Herausforderungen für Arbeitswelt und Gesellschaft. In: Boes A (Hrsg) Digitalisierung und Dienstleistungen. Campus, Frankfurt/New York, S 16

Davis FD (1985) A technology acceptance model for empirically testing new end-user information systems: theory and results. Diss. Massachusetts Institute of Technology, MIT Sloan School Management, Cambridge, MA

DeLone WH, McLean ER (1992) Information systems success: the quest for the dependent variable. Inf Syst Res 3(1):60-95

Destatis (2019a) Kindeswohlgefährdungen 2018: Jugendämter melden 10\% mehr Fälle - Rund 50400 betroffene Kinder und Jugendliche. Pressemitteilung Nr. 337 vom 6. September 2019. Destatis, Wiesbaden

Destatis (2019b) 2018 erstmals über 1 Million erzieherische Hilfen für junge Menschen - Inanspruchnahme innerhalb von zehn Jahren um gut ein Viertel gestiegen. Pressemitteilung Nr. 424 vom 31. Oktober 2019. Destatis, Wiesbaden

Flick U (2011) Triangulation: Eine Einführung. Qualitative Sozialforschung. VS, Wiesbaden

Fließ S, Dyck S, Schmelter M, Volkers MJ (2015) Kundenaktivitäten in Dienstleistungsprozessen - die Sicht der Konsumenten. In: Fließ S, Haase M, Jacob F, Ehret M (Hrsg) Kundenintegration und Leistungslehre. Springer, Wiesbaden, S 181-204

Helming E, Blüml H, Schattner H (1999) Handbuch Sozialpädagogische Familienhilfe. Bundesministerium für Familie, Senioren, Frauen und Jugend Schriftenreihe, Bd. 182. Kohlhammer, Stuttgart

Herterich M, Uebernickel F, Brenner W (2016) Industrielle Dienstleistungen 4.0. Springer, Wiesbaden, S 2

Lantow B, Fellmann M, Lambusch F, Simon G (2020) Requirements for IT-support of personal services in the digital age. In: Zimmermann A, Schmidt R, Lakhmi CJ (Hrsg) Architecting the digital transformation. Springer, Heidelberg Berlin (In Veröffentlichung)

Motahari-Nezhad HR, Swenson KD (2013) Adaptive case management: overview and research challenges. In: Conference on business Informatics Wien, S 264 
Oelerich G, Otto H-U (2011) Empirische Forschung und Soziale Arbeit: Ein Studienbuch. VS, Wiesbaden Schickler M, Reichert M, Pryss R, Schobel J, Schlee W, Langguth B (2015) Entwicklung mobiler Apps: Konzepte, Anwendungsbausteine und Werkzeuge im Business und E-Health. Springer Vieweg, Wiesbaden, $\mathrm{S} 17$

Schütte G (2014) Dienstleistung in der digitalen Gesellschaft - Grußwort des Staatssekretärs im Bundesministerium für Bildung und Forschung. In: Boes A (Hrsg) Dienstleistung in der digitalen Gesellschaft: Beiträge zur Dienstleistungstagung des BMWF im Wirtschaftsjahr 2014. Campus, Frankfurt/New York, S 11

Simon FB, Clement U, Stierlin H (1999) Die Sprache der Familientherapie: ein Vokabular; kritischer Überblick und Integration systemtherapeutischer Begriffe, Konzepte und Methoden. Klett-Cotta, Stuttgart, S 117

Sonnenberg C, v. Brocke J (2012) Evaluations in the science of the artificial-reconsidering the buildevaluate pattern in design science research. In: Peffers K, Rothenberger M, Kuechler B (Hrsg) Design science research in information systems: advances in theory and practice. Springer, Berlin/ Heidelberg, S 392

Statista (2019) Ausgaben für Kinder- und Jugendhilfe in Deutschland nach Verwendungszweck im Jahr 2017 (in Millionen Euro). https://de.statista.com/statistik/daten/studie/71985/umfrage/ausgabenfuer-kinder--und-jugendhilfe/. Zugegriffen: 11. Juni 2020

Zühlke D (2011) Nutzergerechte Entwicklung von Mensch-Maschine-Systemen: Useware-Engineering für technische Systeme. Springer VDI, Düsseldorf, S 85 